Radiologe 2022 · 62:1-2

https://doi.org/10.1007/s00117-021-00949-6

Angenommen: 1. Dezember 2021

(c) The Author(s), under exclusive licence to Springer Medizin Verlag $\mathrm{GmbH}$, ein Teil von Springer Nature 2021

\section{Wissenschaft und Wirklichkeit}

\author{
S. Delorme ${ }^{1} \cdot$ J. Hillengass ${ }^{2}$ \\ ${ }^{1}$ Abteilung Radiologie (E010), Deutsches Krebsforschungszentrum, Heidelberg, Deutschland \\ ${ }^{2}$ Department of Medicine, Roswell Park Comprehensive Cancer Center, Buffalo, USA
}

\section{Autor}
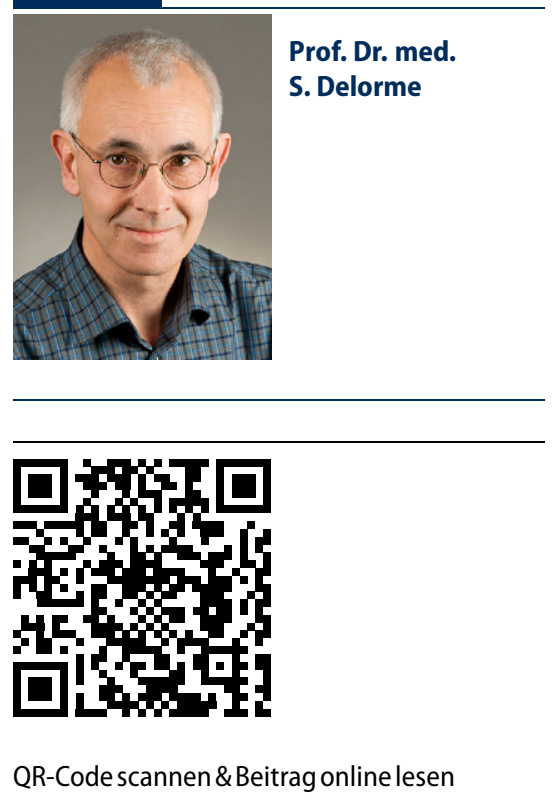

Liebe Leserinnen und Leser,

die Fortschritte der Behandlung des multiplen Myeloms in den vergangenen Jahren sind beeindruckend. Zugleich verzeichnen wir zunehmend eine Individualisierung der Therapieindikationen, u.a. auf der Grundlage der zytogenetischen Charakterisierung, aber auch anhand von Befunden bildgebender Verfahren. Diese ihrerseits haben zum einen technische Entwicklungen erfahren, zum anderen ist ihr Beitrag zur Abschätzung des Progressionsrisikos empirisch so gut abgesichert, dass auf ihrer Grundlage klinische Entscheidungen getroffen werden können.

Vorbei sind die Zeiten, da der Alternator mit Skelettaufnahmen vollgehängt wurde, der Radiologe achtgeben musste, in deren Flut nicht die Übersicht zu verlieren, und rätseln musste, ob eine Aufhellung nun im Knochen, oder irgendwo davor oder dahinter im Strahlengang lokalisiert war. Passé auch die Zeit, als sich das Knochenmark, der Ursprung der Erkrankung, der bildgebenden Diagnostikentzog, denn das Skelettröntgen zeigt nur, was das multiple Myelom am mineralisierten Knochen angerichtet hat. Und auch das nur unzureichend. Dass die Ganzkörper-Computertomographie (CT) ihm darin überlegen ist, ist belegt. Zudem zeigt sich auch, dass die in der CT sichtbaren Befunde, die dem Röntgen entgehen, prognostisch relevant sind.

Die Standards moderner Bildgebung des multiplen Myeloms stehen einstweilen fest: Computertomographie, Magnetresonanztomographie (MRT) und Positronenemissionstomographie (PET) - jede Methode mit ihren Stärken und Schwächen. Wann was wie gemacht werden sollte, kann man nachlesen, so z. B. in den Leitlinien der International Myeloma Working Group (IMWG) und inzwischen auch der Deutschen Krebsgesellschaft. Mit der Umsetzung hingegen hapert es noch. Gewiss spielen Kosten und Verfügbarkeit eine Rolle, aber auch die Trägheit des Systems, das sich manchmal schwertut, selbst offensichtliche Verbesserungen umzusetzen. Dies schlägt sich auch, leider, in den Leitlinien nieder. Noch mehr als die nationalen sind internationale Leitlinien bisweilen von ökonomischen Faktoren und Problemen der Verfügbarkeit geprägt. So wurde zum Beispiel in der Bildgebungsleitlinie der IMWG die Ganzkörper-CT für die Diagnostik in frühen Stadien der MRT vorgezogen, da diese im Unterschied zu Deutschland in vielen anderen Ländern nur sehr spärlich zur Verfügung steht. So widersinnig dies ist, erschwert der Verweis auf die IMWG-Leitlinie die Implementierung der MRT in Deutschland, wo die Versorgung mit MRT-Scannern eigentlich gut ist. Bei der Verhinderung einer Methode zumindest funktioniert dieses Spiel, leider nicht bei der Förderung: So bewegt sich trotz der Empfehlung der IMWG zum Einsatz der PET/CT in Deutschland dazu nicht viel: Ein paar verschämte "kann"Empfehlungen, versehen mit Caveats wegen der ungesicherten Vergütung. Dies ist der Leitliniengruppe nicht anzulasten; eine stärkere Empfehlung hätte wenig Sinn gehabt, solange man von der Methode fordert, dass ihre Anwendung oder Nichtanwendung auf Endpunkte einwirkt, die in ferner Zukunft liegen. Dieses Schicksal teilt auch die Ganzkörper-MRT, die in der Logik der gesetzlichen Krankenkassen, man höre und staune, ein „neues Verfahren“ darstellt, das somit in Hinblick auf die Vergütung 
überhaupt nicht existiert. Somit müsste die Ganzkörper-MRT erst einmal eine formelle Methodenbewertung durchlaufen, was ohne Weiteres ein gutes Jahrzehnt benötigen kann. So, als hätte man eine neue Maschine gebaut.

Doch lassen wir das Zagen, und staunen wir stattdessen darüber, was erreicht wurde: Mit allen drei Methoden ist eine Aussage über das Knochenmark und einen extramedullären Myelombefall möglich. Die MRT einschließlich der Diffusionsbildgebung erfasst, wie Myelomzellen im Knochenmark wohnen oder darin eigene Nester entwickeln - in diesem Heft aber erfahren wir, was der Pathologe auf mikroskopischer Ebene sieht (s. Beitrag von A. Vogelsberg et al.). Die PET/CT berichtet über die Aktivität und Vitalität des Myeloms, wie es auf eine Therapie anspricht, bei welchen Patienten wir mit einem frühen Rezidiv rechnen müssen, und bei welchen möglicherweise eine bildgebend nachweisbare Resterkrankung vorliegt. In diesem Heft erfahren wir, wie wir als Radiologen mit PET/CT-Bildern umgehen und sie analysieren können (s. Beitrag von C. Sachpekidis et al.). Wir können lesen, wie heute eine moderne Therapie des multiplen Myeloms aussieht, wohin die Entwicklung noch gehen könnte (s. Beitrag von H. Goldschmidt) und wann und wie eine Strahlentherapie die systemische Therapie ergänzt (s. Beitrag von L. König und K. Herfarth). Schließlich haben wir der neuen AWMFLeitlinie einen Beitrag gewidmet, genauer dem, was darin für die bildgebende Diagnostik für die nächste Zeit maßgeblich ist (s. Beitrag von M.-A. Weber und A. Baur-Melnyk). Zuletzt: Kaum ein Kongress, bei dem nicht zumindest eine Veranstaltung auch den Möglichkeiten von Radiomics und der künstlichen Intelligenz (KI) gewidmet ist. Allerdings sind beim multiplen Myelom die Herausforderungen gewaltig, will man nicht eine "region of interest", um einen willkürlich gewählten Herd malen, oder in irgendeine Region des Knochenmarks, die man für repräsentativ hält. Hier hat sich einer der Sisyphosarbeit angenommen, der KI beizubringen, wie sie das gesamte Knochenmark erkennen und segmentieren kann (s. Beitrag von M. Wennmann und J. M. Murray). Ist dies erst geschafft, kann die KI ihre eigentliche Arbeit aufnehmen.
Trotz aller Anstrengungen und Fortschritte ist das multiple Myelom nicht heilbar. Wer immer die Diagnose eines symptomatischen multiplen Myeloms einmal hat, ob high- oder low-risk, wird vermutlich daran sterben, sofern dem nicht eine andere Todesursache zuvorkommt. In hohem Alter kann dies einer normalen Lebenserwartung entsprechen, aber wen die Krankheit in mittleren oder gar jungen Jahren trifft, sieht einem langen, teilweise schmerzvollen und am Ende unaufhaltsamen Leiden entgegen. Trotz aller Erfolge überlebt noch immer irgendeine maligne Zelle, die sich allen Einflüssen entzogen hat, und bereit steht für den nächsten, vielleicht tödlichen Schub. Zu erwarten, dass bildgebende Verfahren hieran etwas ausrichten könnten, mag vermessen sein. Gleichwohl unterstützen sie die klinischen Kolleginnen und Kollegen in gut fundierten Entscheidungen und tragen dazu bei, dass Kranke die Behandlung erhalten, die sinnvoll und wirksam ist, und auch nur diese. Voraussetzung hierfür ist, dass die Verfahren auf technisch aktuellem Stand betrieben werden, dass Onkologie und Radiologie einen lebendigen Dialog pflegen, durch den ihr Fachwissen und ihre Entscheidungen reifen können.

Ihre

Stefan Delorme

Jens Hillengass

Korrespondenzadresse

Prof. Dr. med. S. Delorme

Abteilung Radiologie (E010), Deutsches

Krebsforschungszentrum

Im Neuenheimer Feld 280, 69120 Heidelberg,

Deutschland

s.delorme@dkfz-heidelberg.de

Interessenkonflikt. S. Delorme und J. Hillengass geben an, dass kein Interessenkonflikt besteht.
MED UPDATE SEMINARE 2022

Radio-Onko Update 2022

6. Radioonkologie-Update-Seminar

18.-19. November 2022

Berlin und Livestream

Wiss. Leitung:

Prof. Dr. Simone Marnitz-Schulze, Köln

Prof. Dr. Stephanie E. Combs, München

Prof. Dr. Cordula Petersen, Hamburg

www.radio-onko-update.com

Auskunft für alle Update-Seminare:

med update $\mathrm{GmbH}$

www.med-update.com

Tel.: 0611 - 736580

info@med-update.com 